\title{
La cosmicité de l'existence : entre Patočka et Minkowski
}

Jean-Claude Gens

\section{(2) OpenEdition}

1 Journals

Édition électronique

URL : https://journals.openedition.org/alter/1862

DOI : $10.4000 /$ alter. 1862

ISSN : 2558-7927

Éditeur :

Association ALTER, Archives Husserl (CNRS-UMR 8547)

\section{Édition imprimée}

Date de publication : 1 novembre 2019

Pagination : $29-45$

ISBN : 978-2-9550449-5-7

ISSN : $1249-8947$

\section{Référence électronique}

Jean-Claude Gens, "La cosmicité de l'existence : entre Patočka et Minkowski », Alter [En ligne], 27 |

2019, mis en ligne le 22 décembre 2020, consulté le 13 juin 2021. URL : http://

journals.openedition.org/alter/1862 ; DOI : https://doi.org/10.4000/alter.1862

Ce document a été généré automatiquement le 13 juin 2021

Revue Alter 


\title{
La cosmicité de l'existence : entre Patočka et Minkowski
}

\author{
Jean-Claude Gens
}

1 La convergence de deux dimensions des pensées de Patočka et de Minkowski en direction d'une phénoménologie non-égologique de la vie permet d'envisager la possibilité non tant d'une « comparaison » qui conduit bien souvent à juxtaposer des pensées de façon assez stérile, mais de lire Patočka à partir de Minkowski et inversement. S'ils se sont tous deux recommandés d'une phénoménologie dont le sens est assez différent, ils ont tous deux invité à reconnaître, plus, à interroger le caractère mouvant et en devenir d'une dimension d'où sont susceptibles de se constituer des cosmos et d'émerger des égos ; leurs pensées convergent en second lieu au regard de la nature en fin de compte pratique, éthique et/ou politique de leurs visées. Après quelques considérations historiques préliminaires, j'en viendrai à la cosmologie vers laquelle pointent les deux phénoménologues, puis à l'étrange écart qui sépare leurs conceptions pourtant analogues des différents mouvements de l'existence - un écart qui sera questionné au regard de ce que furent les expériences décisives de leurs propres existences; un dernier temps, plus bref, s'arrêtera à la dimension éthique de leurs pensées et, à titre de conclusion, sur la consistance de la philosophie de l'histoire de Patočka.

2 Minkowski (1875-1972) et Patočka (1907-1977) ne sont pas de la même génération. 1936 voit la publication simultanée de Vers une cosmologie, le dernier ouvrage de la trilogie minkowskiennne, et du premier livre de Patočka: Le monde naturel comme problème philosophique. Il n'y a pourtant pas tellement de traces indiquant qu'ils se soient lus ${ }^{1}$. S'ils se sont tous deux recommandés de la phénoménologie, leur phénoménologie n'est certes pas identique, celle dont Minkowski se réclame, c'est celle du premier Husserl, de Max Scheler et des phénoménologues du cercle de Munich où il suit, entre 1910 et 1914, les cours d'Alexander Pfeiffer et de Moritz Geiger. Dans le numéro de la Revue Internationale de Philosophie du 15 octobre 1939 qui publiait son « Naître et prendre naissance $»^{2}$ dont il sera plus loin question, Minkowski a probablement vu la mention 
très lapidaire de la parution du Edmund Husserl, zum Gedächtnis de Landgrebe et de Patočka.

3 De son côté, si Patočka se réfère bien à «Minkowski » à propos de la question de l'espace, ce n'est pas, pour ainsi dire, le bon, puisque c'est Hermann le physicien et non Eugène le psychiatre phénoménologue ${ }^{3}$. Il a en revanche pu voir la référence à plusieurs reprises à Minkowski de la Phénoménologie de la perception de Merleau-Ponty, et peut-être aussi les références à Minkowski de son ami Binswanger ${ }^{4}$ avec lequel il fonde la psychiatrie phénoménologique au congrès de Zurich en 1922. Pour construire le dialogue que les deux philosophes auraient pu avoir, il serait possible de partir de leur réception en particulier de Bergson - une réception tout à fait décisive en ce qui concerne le psychiatre - et de Heidegger, dont Minkowski sait aussi combien sa pensée a marqué l'élaboration de la Daseinsanalyse de Binswanger ${ }^{5}$, mais dont lui-même se démarque.

4 Mais l'absence de références réciproques et la difficulté à croiser leurs réceptions de Bergson et de Heidegger est peut-être plus une chance qu'un défaut. Car ce genre d'approche reconduirait trop facilement à une histoire antiquaire de la philosophie, certes nécessaire, mais d'un intérêt par avance limité, en particulier dans la mesure où elle serait décalée par rapport à l'allure très libre et légère du style de Minkowski qui cherche à décrire des expériences vives sans s'encombrer ni d'une quelconque terminologie scolastique aussi "phénoménologique» soit-elle, ni de références académiques, c'est-à-dire sans se sentir obligé de se démarquer à chaque fois vis-à-vis de ce que ses prédécesseurs ont pensé. Il me semble en revanche bien plus conforme à leurs pensées d'en interroger la teneur en mettant en évidence les expériences qui ont décidé de la direction cosmologique de ces pensées - une direction qui n'est pas non plus sans parenté avec celle prise par le dernier Merleau-Ponty.

5 Les conceptualités de Minkowski et de Patočka sont assez différentes ne serait-ce que parce que Minkowski ne réinvestit pas les concepts des phénoménologies husserlienne et heideggérienne; il ne dit ainsi rien quant à la nature, par exemple, du transcendantal, de l'épokhè ou de la réduction..., et, dans ses écrits, on ne trouverait pas de considérations relatives aux "structures de l'apparaître", aux modalités de « l'apparaître du monde »... Mais Minkowski cherche à " saisir sur le vif », comme il dit, le déploiement de l'existence dans une mobilité qui est, comme on le verra, simultanément celle du monde et de l'homme. Par ses descriptions de ce déploiement, y compris lorsque celui-ci prend des formes pathologiques - car Minkowski pense sur l'arrière-plan de son expérience de psychiatre -, il est bien plus phénoménologue que bergsonien lorsqu'il se borne à décrire une expérience pourtant, pour ainsi dire, interdite par le cadre conceptuel traditionnel et dominant, celui propre, en particulier, au dualisme moderne, sans succomber à la tentation d'une nouvelle théorisation qui menacerait d'éteindre ou d'étouffer cette expérience vive. Avoir surmonté le dualisme moderne impliquerait de ne plus être surpris par l'éventualité qu'un psychiatre se propose de penser une cosmologie et de mettre ainsi en évidence notre cosmicité. Qu'en est-il donc de cette cosmologie?

6 Les pensées de Patočka et de Minkowski visent toutes deux à dé-couvrir la mobilité ou le mouvement en commençant par libérer cette notion de sa réduction moderne à la kinesis, au pur transport. C'est ce que disent déjà les expressions patočkiennes de mouvements de la vie, de mouvement de l'existence, et la notion minkowskienne de 
«mouvement formatif », comme la définition de la cosmologie en tant que son objet est constitué par « les mouvements communs à l'âme et à la nature "

7 L'idée d'une telle cosmologie et de ces mouvements communs impliquent de dépasser non seulement le dualisme entre l'âme ou l'esprit et la nature, mais l'idée même de corrélation intentionnelle husserlienne en tant qu'elle s'inscrirait dans une égologie. Vers une cosmologie critique en ce sens la distinction entre espaces et mouvements " physiques » et " psychiques ", en particulier dans un chapitre où Minkowski procède à une description phénoménologique de l'expérience que tout un chacun a pu faire en accompagnant un proche à la gare. Sa description met en évidence le caractère artificiel d'une distinction entre un accompagnement "physique " lorsque, le train ébranlé et commençant à rouler, on l'accompagne en marchant sur le quai de la gare parallèlement à ce train d'une part, et, le train disparu, on l'accompagne par la pensée d'autre part, car il n'y a en réalité qu'une seule et unique expérience ${ }^{7}$.

Minkowski décrira ainsi des mobilités au sujet desquelles il n'y a pas de sens à se demander si elles sont physiques ou psychiques, en forgeant pour ainsi dire de nouveaux concepts comme se répandre, remplir, retentir (des catégories donc, d'une mobilité vivante) ; partant ainsi du phénomène du retentissement, Minkowski redéfinit ainsi la cosmologie qu'il appelle de ses vœux :

Tout autour de nous le monde retentit de mille mélodies, exhale mille parfums s'anime de mille mouvements qui font tressaillir et palpiter tout notre être, et nous participons à cette vie [...] à laquelle nous venons puiser nos forces et notre raison d'être, que nous voudrions aussi connaitre, en cherchant à saisir sur le vif [...] les qualités dynamiques dont elle se compose [...]. C'est là le but que poursuit la cosmologie. (VC, $171 \mathrm{sq}$.)

La cosmologie telle que la pense Minkowski vise donc à décrire la dimension dynamique - qu'il appelle parfois nature mais le plus souvent vie - dans laquelle adviennent l'homme et le monde. Dans l'avant-propos du Temps vécu, Minkowski pointe la commune origine, à ses yeux, des philosophies de Husserl et de Bergson : l'intention de rétrocéder en amont du mouvement des sciences et des techniques en direction de " la nature », c'est-à-dire "[...] reprendre contact avec la vie et ce qu'il y a de "naturel" et de primitif en elle, revenir vers la source première dont jaillissent non seulement la science, mais aussi toutes les autres manifestations de la vie spirituelle » (TV, 2 sq.).

Vers une cosmologie invite en ce sens à substituer à l'anthropologie classique, qui manque l'humain du fait de son anthropocentrisme, ce qu'il appelle - d'un terme que l'on retrouvera plus tard, par exemple, dans Images et symboles de Mircea Eliade - un "anthropocosmisme $»^{8}$. C'est encore cette dimension anthropo-cosmique de notre vie qui est pointée dans le passage suivant de Vers une cosmologie : " "humain" ne veut point dire "propre à l'homme"; loin de là, il le déborde largement, pour se confondre avec l'univers » $(\mathrm{VC}, 150)$.

11 Minkowski reviendra encore sur cet anthropocosmisme dans une intervention au Congrès International de Philosophie à Amsterdam en 1948 - une intervention intitulée «Le contact humain ». Il y invite à penser l'anthropologie non seulement dans le cadre d'un anthropocosmisme, mais encore ce qui en constitue à son sens le versant complémentaire : celui qu'il qualifie de cosmo-anthropologique. Se référant sans le nommer à Heidegger en écrivant "l'homme est dans le monde, c'est entendu ", Minkowski suggère que si le premier versant concerne la manière dont l'homme est "dans le monde ", le second interroge la manière dont le monde «le reçoit en son 
sein ", ce qui, de ce monde, pénètre en lui pour en former l'essence ${ }^{9}$ - la suite du texte faisant à nouveau usage du terme de nature. Ces deux versants disent le double mouvement d'insertion dans, et d'accueil de, l'élan vital vécu. Mais ce double mouvement se double, comme on le verra d'un « mouvement oscillatoire ${ }^{10}$ bien plus essentiel et, pour ainsi dire, vertical entre deux plans de cet élan vital.

De ce point de vue, ce n'est pas tant la révision radicale, par Patočka, de la notion husserlienne de monde naturel - à propos duquel il écrit : si «nous nous tournons vers lui, [c'est] parce que nous cherchons la vie dans son originalité $»^{11}-$ qui, pour ainsi dire, répond à Minkowski, mais son réinvestissement de la notion grecque de phusis. En ce qui concerne la première de ces deux notions, la critique de la notion husserlienne de monde naturel, ou de monde de la vie, tient à l'idée qu'il nous faut pouvoir penser un monde « où on doit pouvoir vivre, vivre en commun, où on doit être accepté et reçu »" Autrement dit, « le monde naturel doit être considéré en dernière analyse comme celui de l'historicité concrète, non seulement du fait de sa traditionnalité essentielle, mais en raison du drame, de "l'histoire" qui s'y déroule $»^{13}$ - une histoire qui n'est pas moins naturelle qu'historique...

13 Renvoyant par ailleurs à Fink, Patočka invite encore à penser en termes de phusis le fond à partir duquel se déploie l'individuation conjointe de l'homme et de ses mondes de la vie ; cette phusis est :

Le cadre préalable, total, non individué, de toute individuation. Indivis, c'est lui qui à la fois sépare et relie tout [...]. La manifestation des choses rendue possible par ce cadre n'est pas manifestation pour le sujet, mais bien manifestation comme entrée dans la singularité, devenir (MNMEH, 100).

14 Autrement dit, c'est de ce tout du monde non individué, qui n'est "pas" une individualité dont émergent des individualités en se distinguant les unes par rapport aux autres et en s'opposant ainsi les unes aux autres (MNMEH, 269). Patočka avance ainsi :

La philosophie propose un nouveau visage de l'impérissable - non plus seulement la permanence, l'immortalité, la continuité propres aux dieux - mais l'éternité qui se présente tout d'abord à elle sous la forme de la phusis dont procèdent l'éclosion et l'éclipse de tout étant, son émergence, sa croissance et son déclin, son engloutissement dans les ténèbres. À la nuit de la phusis appartient l'aube du cosmos, l'aube de l'ordre des choses comme ce qui, loin d'atténuer le mystère de l'être et des étants, l'accentue (EH, 90).

Si l'interrogation de Patočka relèverait en ce sens d'une "cosmologie phénoménologique », Minkowski suggère de son côté de penser cette émergence ou cette individuation non pas en termes de naissance, mais d'un prendre naissance dont il met en évidence la signification dans un article intitulé " Naître et prendre naissance » (1939). Parue trois ans après Vers une cosmologie dans le dernier numéro avant la Seconde Guerre de la Revue Internationale de Philosophie - Minkowski s'y trouve en très bonne compagnie, puisque y contribuent aussi Jankélévitch, Lavelle, Le Senne ou Gabriel Marcel -, la force de cette contribution à une possible phénoménologie de la naissance ou de la natalité tient à la simplicité de son geste. Dans une perspective que l'on pourrait comparer à celle de Heidegger ou de Hegel qui font en quelque manière parler la langue allemande, Minkowski suit au plus près les tournures usuelles de la langue française en considérant l'expression prendre naissance comme il le faisait à propos du prendre corps dans Vers une cosmologie (VC, 156 sqq., 262) pour désigner la 
manière dont le passage de l'obscurité à la clarté voit progressivement se dessiner et s'affirmer les contours déterminés des corps ou des idées.

Minkowski distingue ainsi d'une part la naissance qui a un début et une fin et s'inscrit dans une temporalité où il $\mathrm{y}$ a des avants et des après, par exemple notre naissance telle qu'elle est enregistrée par l'état civil, et d'autre part un prendre naissance. Par exemple, un torrent qui prend naissance dans un glacier sans qu'il soit possible de repérer un début et une fin : «Le fleuve renouvelle à chaque instant, sans discontinuer, ses eaux. C'est un renouveau éternel, lourd de tout un passé, mais toujours jeune, ne s'épuisant jamais, ne connaissant point la monotonie ${ }^{14}$.

17 Pour nommer ce prendre naissance comme le donner naissance ${ }^{15}$, qui « ne se déroule pas dans le temps» et ne s'épuise jamais, Minkowski propose aussi l'expression de " mouvement formatif $»^{16}$. La cosmologie qui intéresse ainsi Minkowski ne décrit aucun événement passé, aucune origine historique ou préhistorique au sens usuel de ces termes, mais le présent constant de la vie en son jaillissement, son mouvement perpétuel.

18 À l'occasion d'une relecture d'Erwin Straus et de Viktor von Gebsattel à propos de la psychose maniaco-dépressive (TV, 277 sq.), Le temps vécu décrit encore, sans le nommer ainsi, le mouvement complémentaire du prendre fin. Il distingue entre la mort immanente et la mort transitive. Si la mort transitive, « en force étrangère et hostile, vient du dehors détruire brutalement notre vie» (TV, 284), en revanche la mort immanente "intimement liée à la vie, la suit comme son ombre», car "nous ne marchons pas vers elle, mais c'est elle, au contraire, qui nous suit pas à pas, en compagnon fidèle » (TV, 284), dans la mesure où l'accomplissement de toute œuvre, l'apogée de tout sentiment, marque une fin et un autre début. Cette mort advient donc sans cesse, c'est-à-dire advient en dehors du temps. Et au fur et à mesure que la vie avance, le poids de ces achèvements croît, lorsque «notre vie semble avoir réalisé ce qu'il lui appartenait de réaliser, la mort met fin à notre devenir individuel » (TV, 284). Le statut de cette mort est en ce sens singulier, car si elle ne rompt pas brutalement, comme la mort transitive, le cours de l'existence; elle est immanente à la vie, tout en mettant fin à son devenir...

19 Il convient néanmoins de revenir au " Naître et prendre naissance » où se dessine pour ainsi dire la cosmologie phénoménologique patočkienne, si l'on entendait par là la tentative de décrire la manière dont naissent conjointement, à partir du fond de la phusis, un ou des cosmos et l'homme en lequel le cosmos s'apparaîtrait ${ }^{17}$. Le prendre naissance, écrit Minkowski, nomme effectivement aussi le processus de l'écriture d'un livre ou de l'émergence d'une œuvre d'art, et plus largement de nos vies en leur humanité; si ce prendre naissance implique une durée comme tout prendre, comme lorsqu'on dit ça prend, l'élan créateur, qui « vient du fond de notre être ", prend ainsi naissance « au-delà, dans la vie ». Ou, pour le dire autrement : «L'homme appelé à vivre une vie humaine prend naissance ailleurs qu'il ne naît $»^{18}$.

20 Et l'au-delà de la vie des mondes de la vie, l'ailleurs de la naissance, Minkowski invite à le penser comme un dynamisme dans lequel ne se distingue initialement aucune forme précise, une plénitude «de mystère, de promesses, un dynamisme générateur de cosmos et de nous-mêmes ${ }^{19}$, un néant, « source de toutes choses $»^{20}$ dans lequel «la vie humaine se retrempe toujours à nouveau pour y prendre toujours à nouveau naissance, pour s'y forger dans un mouvement éternel $»^{21}$ : participation à une vie cosmique éternelle. Il est à mon sens difficile de ne pas penser ici à la manière très 
similaire dont Patočka invite à penser le néant en lequel s'originent l'homme et le cosmos, par exemple lorsqu'il demande: "Ne persiste-t-on pas [...] à méconnaître la clôture originelle de l'étant sur soi-même, la non individuation originelle, la nuit obscure de l'être-étant? » (MNMEH, 99). Qu'en est-il plus précisément de la mobilité de la vie cosmique?

La convergence entre les cosmologies patočkienne et minkowskienne s'atteste encore en ce qui concerne la manière dont la mobilité de notre vie ou de notre existence est pensée. À la distinction entre les trois mouvements de l'existence chez Patočka, les mouvements d'acceptation, de défense - ou de dessaisissement de soi impliqués par la nécessité de travailler - et de vérité ( $\mathrm{EH}, 51 \mathrm{sq}$.) (ou d'enracinement, de reproduction et de prolongement de soi, et enfin de percée) (MNMEH, 101-124) - qui se déploient avec un accent temporel singulier, répondent les mouvements qui, dans la pensée de Minkowski, caractérisent la vie en sa vitalité : 1) le contact vital avec la réalité - forgé à partir de la notion bleulerienne de syntonie, et plus largement dans le cadre d'une confrontation avec la schizophrénie, qui est plus qu'un « contact » puisqu'il relève d'un mouvement de sympathie par lequel le vivant participe de la nature fluide du devenir $^{22}$; 2) le mouvement par lequel il nous faut nous en arracher pour pouvoir satisfaire au désir de marquer le devenir de notre "empreinte personnelle", d'« imposer notre moi au monde infini » (TV, 69), ce qui implique non seulement une séparation, mais d'entrer en conflit avec d'autres formes de vie; et enfin 3) l'élan créateur et éthique $(\mathrm{VC}, 200)$ qui relève comme d'une « vie dans la vérité » comme dit Patočka, de « cette vie humaine [qui] se meut entre les deux pôles que sont la vérité et la non-vérité $\aleph^{23}$, pour autant que cette vérité soit entendue sur un plan concret, c'està-dire éthique, et qu'elle soit susceptible d'appeler au sacrifice de soi (TV, 109) - « toute la vie psychique étant traversée d'un mouvement oscillatoire " qui nous fait ou est susceptible de nous faire passer d'un plan à l'autre, du plan vital au plan qui nous élève au-delà de la vie ${ }^{24}$.

La temporalité propre à ces mouvements est, là encore, du même ordre : d'un côté le mouvement d'acceptation est relatif à ce qui est déjà là, alors que la confrontation avec les choses et les autres hommes concerne le présent et que l'avenir est l'élément « dans et à partir duquel nous vivons » dans le troisième mouvement (MNMEH, 264). Du côté de Minkowski, le contact vital avec la réalité a pour arrière-plan une nostalgie irréductible, l'affirmation de soi régit l'activité présente, et l'avenir est ouvert par l'action éthique (TV, 103).

En des termes différents, ceux de terre pour Patočka et de vie pour Minkowski, tous deux marquent la spécificité du troisième mouvement par rapport aux deux premiers. "Dans les deux premiers mouvements, la terre règne", alors que le "mouvement de percée " "bat en brèche le terrestre ", ébranle "la terre en nous » ${ }^{25}$, de même le contact vital et la nécessité de se démarquer de la vie comme tout indistinct sont les deux aspects qui caractérisent le cycle de «l'élan personnel» (TV, 69), dont Vers une cosmologie invite à penser la contradiction en termes de « conflit anthropocosmique ${ }^{26}$, alors que l'action éthique "plane au-dessus de la vie» (TV, 107). Les différences entre ces trois mouvements ne sont pourtant pas moins patentes que leurs analogies. Ainsi, le contact vital avec la réalité n'est pas identique au mouvement de l'acceptation qui est immédiatement d'ordre plus intersubjectif et qui renvoie à l'accueil, au foyer... (MNMEH, 37) De même, marquer le devenir de son empreinte, chercher l'affirmation du moi (VC, 198 et 197), ce n'est pas seulement devoir lutter et travailler. 

154 sq., 159, 161) en se référant à Teilhard de Chardin ${ }^{31}$ et à Ernst Jünger mais qu'il n'a pas pu faire directement, c'est celle du front. Cette expérience rejette les déterminations sociales et culturelles à l'arrière-plan pour reconduire à l'existence pour laisser émerger une intersubjectivité et une intercorporéité plus primitives :

La découverte la plus profonde du front, cette avancée de la vie dans la nuit, le combat et la mort, l'impossibilité de rayer de la vie ce paragraphe qui, du point de vue du jour, apparait comme pure et simple non-existence ; c'est la transformation du sens de la vie qui se heurte ici au néant, à la frontière infranchissable qui est la figure ultime de tout (EH, 167).

yer à la confrontation de Patočka, à l'histoire pour ainsi dire « objective » ne suffit pourtant pas à expliquer la centralité de la question de l'histoire dans sa pensée ; c'est ce que suggère inversement la déclaration d'un personnage du Livre du rire et de l'oubli de son compatriote Milan Kundera au moment où, dans ce roman, les chars soviétiques entrent dans Prague: "C'est en réalité maman qui a raison: le tank est périssable et la poire est éternelle $»^{32}$, puisque ce qui pour les uns relève du tragique n'appartient pour les autres qu'à l'écume du devenir de la vie naturelle. Est-ce à partir de ce constat

que demanderait à être compris le relatif silence de Minkowski sur l'expérience de ses émigrations successives et sur celle, pour le moins directe, de la Première Guerre ? Il est difficile de le penser puisqu'il est engagé volontaire comme médecin en 1915 et que, envoyé sur le front, il participe aux batailles de la Somme et de Verdun ${ }^{33}$ à l'occasion 
desquelles il a bien dû avoir affaire à ce que l'on a appelé les "gueules cassées » dont le souvenir est aussi préservé par les représentations qu'en a donné Otto Dix, par exemple dans Les joueurs de Skat ou dans Rue de Prague (1920). Il y a pourtant peu de traces évidentes de cette expérience dans les écrits de Minkowski.

L'avant-propos du Temps vécu rappelle que la guerre a profondément modifié sa vie puisque, alors qu'il s'était progressivement éloigné de la médecine depuis 1909, la guerre l'y reconduit et "plus particulièrement à la psychiatrie» (TV, 5). Mais Minkowski voit rétrospectivement dans cette activité de psychiatre ce qui l'a ramené à la vie dans sa concrétude, ce qui a permis de féconder sa pensée philosophique (TV, 6). Et c'est dans ce cadre qu'il forge des notions qui consonnent avec la pensée de Bergson, comme " le contact vital avec la réalité ». Le temps vécu rappelle aussi la joie primitive éprouvée à mouvoir ses membres pendant le repos qui succédait aux moments de combats (TV, 79), ou le fait que, "pour nos tendances destructrices, une mitrailleuse paraît supérieure à un fusil » (TV, 124). La phénoménologie de la mort à laquelle il a travaillé durant l'hiver $1915-1916^{34}$ n'a pas postérieurement donné lieu à des méditations comparables à celles de Teilhard de Chardin ou de Jünger. Une autre référence à la guerre est plus significative: lorsqu'il s'agit de prendre un exemple d'action éthique, Minkowski écrit à propos d'un soldat lors d'une attaque chimique dans les tranchées :

Son voisin lui dit qu'il croit que son masque n'est pas étanche et qu'il n'en a pas d'autre ; un moment d'hésitation ; un seul ; on sent la mort planer autour ; elle se dresse devant les yeux de notre soldat ; mais ce n'est qu'un moment d'hésitation; il tend sans mot dire son masque à son compagnon. Cette scène n'a pas de témoins, si ce n'est la nuit lugubre et les obus qui sèment la mort (TV, 107 sq.).

S'il me paraît difficile de ne voir dans cet exemple qu'une expérience de pensée, n'estce pas là ce que Patočka a appelé l'expérience de la solidarité des ébranlés et que Minkowski semble avoir encore faite lorsqu'une main lui a été tendue dans une tranchée - une main tendue qu'il a expressément perçue comme l'expression d'une "solidarité " $^{35}$ et qui a décidé de son désir de devenir français. C'est encore à l'émergence de cette solidarité qu'il contribue durant la Seconde Guerre lorsqu'il se rend au Vel' d'Hiv au moment de la rafle pour soutenir la détresse de ses victimes ${ }^{36}$, et lorsqu'il présidera la société O.S.E qui sauvera 2000 enfants juifs de la déportation à laquelle succomberont en revanche trente membres de cette sociétés ${ }^{37}$. En ce qui concerne le chez soi ou le foyer, tout se passe comme si son foyer avait été moins géographique et moins européen - même si l'on pensait à l'idée d'Europe - que d'ordre cosmique...

30 Ces épisodes essentiels de l'existence de Minkowski nous conduisent à une dimension des pensées des deux phénoménologies à laquelle il convient pour finir de s'arrêter même si c'est plus brièvement : la centralité de la dimension éthique.

31 Je reviens à l'exemple de celui qui, dans l'imminence d'une attaque, donne son masque à gaz à un autre soldat :

Son voisin lui dit qu'il croit que son masque n'est pas étanche et qu'il n'en a pas d'autre; un moment d'hésitation; un seul; on sent la mort planer autour; elle se dresse devant les yeux de notre soldat; mais ce n'est qu'un moment d'hésitation; il tend sans mot dire son masque à son compagnon. Cette scène n'a pas de témoins, si ce n'est la nuit lugubre et les obus qui sèment la mort (TV, 107 sq.).

32 Ce récit pointe aux yeux de Minkowski le fait que c'est "le facteur éthique ", la tendance éthique qui donne son sens à la vie, elle est « le pilier principal sur lequel la 
vie repose " (TV, 103). Cette "action» (les guillemets sont de Minkowski) qui est la seule à «résiste(r) au devenir, au devenir qui de ses flots gris menace de tout submerger sur son passage " (TV, 108), relève d'un mouvement d'élévation dont Minkowski éclaire la nature à travers une comparaison entre la rancœur et le pardon ${ }^{38}$, c'est-à-dire aussi d'un mouvement d'abnégation de soi, d'un sacrifice qui s'accompagne d'un sentiment singulier d'allégresse. "Le sacrifice de la vie n'est point toujours une fuite devant le déshonneur » $(\mathrm{TV}, 109)$ avance Minkowski, et, quelques lignes plus loin :

Quant à cette formule que la recherche de l'action éthique, allant jusqu'au sacrifice de la vie, ne se fait qu'en vertu du plaisir qu'elle nous procure, nous ne pouvons pas la prendre au sérieux, tant elle est en désaccord avec ce que nous éprouvons en réalité. Ce sentiment d'allégresse n'est d'ailleurs point un sentiment de plaisir à proprement parler. Il consiste surtout en ce que nous voyons, dans l'action éthique, l'univers tout entier s'ouvrir dans toute sa grandeur devant nous, tandis que nousmêmes, nous participons [...] dans une fusion intime, de cette grandeur (TV, 109).

La consonance évidente de ce sens de l'action éthique avec la conception que s'en fait Patočka mériterait d'être interrogée plus avant, et en particulier la signification qu'ils donnent à la dimension du sacrifice à propos duquel je me contenterai d'indiquer deux passages significatifs des écrits patočkiens.

«L'homme spirituel et l'intellectuel » avancent ainsi dans le cadre d'une méditation relative au $\mathrm{XX}^{\mathrm{e}}$ siècle :

Ce monde cruel dont nous avons fait et dont nous continuons à faire l'expérience, le monde inhumain des guerres mondiales, des bouleversements révolutionnaires [...], ce monde - me semble-t-il - n'est de fait compréhensible que parce que les hommes qui ont fait face à ces terribles catastrophes n'y ont pas succombé de manière purement passive, parce que beaucoup d'entre eux se sont jetés volontairement, voire joyeusement dans les gueules de ces Moloch - comme s'ils se rendaient compte que le monde donné et la vie immédiate ne sont pas tout, qu'on peut les sacrifier et, dans ce sacrifice, entrevoir l'éclair au milieu des ténèbres dont parlait Héraclite ${ }^{39}$.

Le sens de cette joie se dit manifestement par exemple dans un passage du « Séminaire sur l'ère technique » de 1973 qui déclare : « On peut risquer sa vie sans se sacrifier, sans accomplir un sacrifice au sens propre et fort de ce terme, où ce ne sont pas deux étants qui sont mis en balance, mais où émerge quelque chose sans commune mesure avec l'étant $»^{40}$ - un "sans commune mesure " avec la terre ou la vie, pour reprendre les termes respectivement de Patočka dans ses Papiers phénoménologiques et de Minkowski dans Le temps vécu. Et c'est encore ce sans commune mesure que dit le terme de «transsubstantiation » dans « Le monde naturel et la phénoménologie »:

La vie est capable non seulement de se prolonger par le dessaisissement de soi, mais encore de se transsubstantier par le dévouement [...] La force de; la transsubstantiation de la vie est la force d'un amour nouveau, d'un amour qui se dévoue aux autres sans condition (1967) (MNMEH, 45).

Pour conclure, la direction empruntée par les pensées de Minkowski et de Patočka conduit, au-delà d'une phénoménologie égologique, en direction d'un anthropocosmisme ou d'une cosmologie phénoménologique, toutes deux interrogent le mouvement du déploiement de la vie et/ou de l'existence dont l'incarnation la fait participer à la mobilité transsubjective du cosmos ou de la phusis. Tous deux accordent par ailleurs - plus que d'autres, et je pense en particulier à Husserl et à Heidegger - un poids absolument décisif à la dimension éthique verticale de l'exister humain, c'est-àdire de la vie dans la vérité, pour reprendre l'expression de Patočka; tous deux 
semblent avoir concrètement témoigné du sens du sacrifice face aux défis des événements historiques auxquels ils ont été confrontés. Il semble en revanche d'autant plus nécessaire de considérer à nouveau la question de savoir comment s'articulent chez Patočka les deux thèmes du monde naturel et de la cosmologie d'une part et de l'histoire d'autre part, car c'est précisément aussi là où les chemins de pensée des deux phénoménologues semblent diverger. C'est dans cette perspective que, partant d'une pensée comme celle de Minkowski, j'aimerais adresser une double question à Patočka.

On aurait du mal à trouver quelque chose comme une « philosophie de l'histoire » chez Minkowski - pas plus peut-être que chez Bergson - et on pourrait être tenté de penser que ces chemins se séparent conformément à la grande ligne de partage entre les philosophies de l'histoire qui dominent la pensée occidentale depuis le XVIII ${ }^{e}$ d'une part, et les philosophies qui sont pour ainsi dire revenues de cette histoire pour privilégier l'interrogation du soubassement naturel de notre historicité ou de son arrière-plan cosmique, comme le feront le mouvement de l'anthropologie philosophique de l'entre-deux-guerres, Löwith et, ultimement, Merleau-Ponty, d'autre part.

38 L'acception la plus immédiate de ce terme d'histoire désigne bien l'ensemble des événements à l'occasion desquels l'humanité, en des guises variées, se constitue en tant que telle, c'est-à-dire s'individue ; c'est la consistance de cette histoire que Le livre du rire et de l'oubli met en doute. Une telle histoire, dans son fracas, celui de l'expérience du «front », n'a-t-elle pas pour sens de permettre une «transsubstantiation » de la vie, de retrouver un au-delà de la vie ou un ailleurs d'où nous procédons sans cesse et qui relève d'une dimension que l'on pourrait tout autant appeler « trans-histoire »?

Mais l'histoire signifie en second lieu pour Patočka la dimension dans laquelle entrent les hommes lorsqu'ils perçoivent et se confrontent à la problématicité du monde qui leur est donné: une problématicité à laquelle "l'humanité pré-historique se dérobe » (EH, $88 \mathrm{sq}$.$) . Si cette problématicité signifie aussi l'étonnement philosophique à$ l'égard «du fait que l'univers soit » $(\mathrm{EH}, 88)$, alors la dimension de «l'histoire » est assurément présente dans la pensée de Minkowski dans la mesure où il pointe souvent, dans ce qui du monde nous est donné, le mystère. Cette acception de la notion d'histoire, Patočka la trouve manifestement chez Karl Jaspers qui me semble bien plus souvent présent à l'arrière-plan de la pensée patočkienne qu'on ne le reconnaît habituellement. Est, par exemple, aussi jaspersienne l'idée que l'existence implique la culpabilité (MNMEH, 117) et, aux yeux de Jaspers, l'histoire effective de l'humanité commence à l'époque qu'il qualifie d'axiale, l'époque à partir de laquelle l'existence fait l'expérience de situations-limites, c'est-à-dire s'apparaît à elle-même dans sa confrontation avec la transcendance. Le monde historique en ce sens ne s'oppose pas au monde naturel, il n'a rien de supra- ou de sur-naturel, il est le monde naturel devenu problématique. J'en viens à la seconde question relative, elle aussi, à l'histoire.

Si c'est au sein de la vie qui va de soi que le basculement vers

le monde dans sa problématicité est susceptible de se produire, "le monde historique " en ce sens-là (le monde vécu dans ce qu'il a de problématique) n'a pas besoin d'être historique au sens usuel, car il y a bien des événements anhistoriques au sens classique de ce terme, qui font voler en éclats l'évidence du sens de la vie ; par exemple le fait d'être quitté par la personne aimée sans s'y être attendu ou le décès d'un proche, de sorte que les phénomènes se voient vidés de ce qui faisait jusqu'alors leur sens ou, plus encore peut-être, le traumatisme qui ouvre la voie à une forme ou à une autre 
d'aliénation mentale, et je pense à L'Idiot de Dostoïevski, au Prince Michkine que sa compassion pour la démence de Rogojine précipite dans l'hébétude - un événement qui appartiendrait à un monde pas moins «naturel » qu' « historique ».

41 Par ailleurs, pour dire ce à quoi se heurte nécessairement l'esprit dans son désir de comprendre le monde, Patočka emploie non seulement le terme de problématicité mais encore celui de mystère qui est, à ses yeux, irréductible à la problématicité. Le problématique désigne ce dont un sujet ou une conscience s'étonne ou peut s'étonner (il peut ainsi signifier la finitude de ce sujet). En revanche le terme de mystère désigne plutôt ce qui, du manifeste, se soustrait à un savoir et tout particulièrement cela même d'où sort le manifeste. Il me semble que c'est dans un telle perspective que demande à être entendu le passage de "La philosophie de la crise des sciences d'après Edmund Husserl et sa conception d'une phénoménologie du "monde de la vie" » où Patočka écrit à propos du monde naturel dont il vient d'indiquer les deux mouvements que sont l'enracinement et la défense, la lutte appelée par la vie :

Enfin il y a [...] des points de repère tout autrement importants et profonds [...] : le ciel et la terre qui, sous la forme inapparente de ce qui, dans et à travers les choses de la nature, est toujours là, se révèlent finalement comme l'enveloppe omnienglobante du mystère des choses de la vie (MNMEH, 241 - souligné par Patočka).

- ce qui nous renvoie en un sens moins du côté de l'histoire que du côté de son soubassement naturel au sens de la phusis. Autrement dit, quel que soit le sens de cette expression, la philosophie de l'histoire ne demanderait-elle pas à être pensée dans le cadre d'une cosmologie phénoménologique ? N'est-ce pas de ce côté que la pensée de Patočka s'atteste dans toute sa radicalité ?

\section{NOTES}

1. Il est néanmoins remarquable de trouver une assez longue référence à J. Patočka, et, en l'occurrence, à ce qu'il dit du "foyer", dans un article intitulé «Le temps des migrants: les temps de l'exil» paru dans la revue Thérapie familiale de 2002/ 2 (vol. 23) (https:// www.cairn.info/revue-therapie-familiale-2002-2-page-183.htm consulté le 1er avril 2019) et qui est écrit par Eva Hemon qui travaille manifestement au Centre F. Minkowska - un centre de consultation médico-psychologique de Paris ouvert aux migrants et créé par E. Minkowski.

2. E. Minkowski, « Naître et prendre naissance », in Revue Internationale de Philosophie, $\mathrm{n}^{\circ}$ 5, 15 octobre 1939, p. 116-127.

3. Voir J. Patočka, «L'espace et sa problématique ", in Qu'est-ce que la phénoménologie ? trad. par E. Abrams, Grenoble, Millon, 1988, p. 35 et 37.

4. L. Binswanger se réfère ainsi à $\mathrm{E}$. Minkowski par exemple dans «Analyse existentielle et psychothérapie » (1958) et dans «À propos de deux pensées de Pascal trop peu connues sur la symétrie » (1947), in Introduction à l'analyse existentielle, Paris, Minuit, 1971, p. 150 et 228.

5. E. Minkowski, « Le contact humain », in Écrits cliniques, Toulouse, Ed. Eres, 2002, p. 141.

6. E. Minkowski, Vers une cosmologie (1936) - dorénavant noté VC suivi de la page dans le corps du texte -, Paris, Payot \& Rivages, 1999, p. 172. 
7. E. Minkowski, Le temps vécu (1933) - dorénavant cité dans le corps du texte TV suivi de la page -, Paris, Presses Universitaires de France, 1995, p. 69-71.

8. E. Minkowski, « Le contact humain », in Écrits cliniques, op. cit., p. 140 et 141.

9. E. Minkowski, «Le contact humain ", in Écrits cliniques, op. cit., p. 141, et p. 143 et 145 à propos du cosmo-anthropologique.

10. Ibid., p. 147.

11. J. Patočka, Le monde naturel et le mouvement de l'existence humaine - sauf exception, dorénavant MNMEH suivi de la page dans le corps du texte -, Dortrecht, Kluwer Academic Publishers, 1988, p. 101.

12. J. Patočka, «La philosophie de la crise des sciences d'après Edmund Husserl... et la phénoménologie du monde de la vie » (1971), in MNMEH, p. 240 et p. 241.

13. J. Patočka, "Phénoménologie du "monde de la vie" ", in MNMEH, p. 241 ; sur la critique de E. Husserl, voir aussi, par exemple, Liberté et sacrifice, Grenoble, Millon, 1990, p. 196, et Essais hérétiques - dorénavant cité EH suivi de la page -, Lagrasse, Verdier, 1999, p. 19, 29, 31.

14. E. Minkowski, « Naître et prendre naissance », op. cit., p. 117 sq.

15. Ibid., p. 125.

16. Ibid., p. 123.

17. Sur cette expression, voir R. Barbaras, Le mouvement de l'existence, Paris, La transparence, 2007, p. 90 sq.

18. E. Minkowski, « Naître et prendre naissance », op. cit., p. 119.

19. Ibid., p. 120.

20. Ibid., p. 122.

21. Idem.

22. E. Minkowski, La schizophrénie (1928), Paris, Payot, 2002, p. 105 sqq. et TV, 58-64.

23. J. Patočka, « Qu'est-ce que l'existence? » (1969), in MNMEH, 251.

24. E. Minkowski, « Le contact humain », in Écrits cliniques, op. cit., p. 147.

25. J. Patočka, Papiers phénoménologiques, Grenoble, Millon, 1998, p. 110, 112.

26. E. Minkowski, VC, 186, 197 sqq., 201, et « Le contact humain », in Écrits cliniques, op. cit., p. 147.

27. Voir H. Declève, «Patočka et les signes du temps ", in Études phénoménologiques, $\mathrm{n}^{\circ} 1,1985$, p. 6, citant « Erinnerungen an Husserl, in Die Welt des Menschen. Die Welt der Philosophie, Festscchrift für Jan Patočka, Phaenomenologica 1976, 72, p. VII.

28. H. Declève, "Patočka et les signes du temps » in Études phénoménologiques, nº 1, 1985, p. 5 note.

29. «Entretien avec Jan Patočka » (1967), in Jan Patočka. Philosophie, phénoménologie, politique, E. Tassin et M. Richir dir., Grenoble, Millon, 1992, p. 15.

30. J. Patočka, «Erinnerungen an Husserl », in Die Welt des Menschen. Die Welt der Philosophie, Festscchrift für Jan Patočka, Phaenomenologica 1976, 72, p. VII, cité par H. Declève, «Patočka et les signes du temps ", in Études phénoménologiques, $\mathrm{n}^{\circ}$ 1, 1985, p. 6.

31. P. Teilhard de Chardin, Écrits du temps de la guerre, Paris, Seuil, Tome XII, 1975.

32. M. Kundera, Le livre du rire et de l'oubli, Paris, Gallimard, « Folio », 1987, p. 52 sq.

33. Voir E. Minkowski, Au-delà du rationalisme morbide, Paris, L'Harmattan, 1997, p. 237.

34. Voir la postface à l'Au-delà du rationalisme morbide, op. cit.

35. Voir le récit qu'en fait sa fille et que rapporte Allen dans sa postface à Au-delà du rationalisme morbide, op. cit., p. 237 : « Un jour de pluie et de grisaille, papa allait tomber dans l'épaisseur de la boue lorsqu'un poilu accourant lui prend la main et l'aide à l'empêcher de s'enliser. Mon père y voit un symbole de solidarité, la main tendue [...]».

36. E. Minkowski, Au-delà du rationalisme morbide, op. cit., p. 239.

37. Voir la postface du Temps vécu, op. cit., p. 403 sq. et, par ailleurs, ses publications «Du temps de l'étoile jaune » (dont les allocutions des Dr. Bessière, V. Cremer, et de M. Alperine) Paris, A. 
Montourcy Impr., 1945, 46 p., la notice nécrologique relative à Aline-Lucie-Mathilde Silz, née 1900, morte en déportation (dont l'allocution du curé de Brunoy, l'abbé Delagarde), Blois, Impr. de R. Sille, 1947, 23 p., "The Psychology of the Deportees » American OSE Review 4, Summer-Fall 1947, «Les conséquences psychologiques et psychopathologiques de la guerre et du nazisme aspect général du problème ", in Schweitzer Archiv für Neurologie und Psychiatrie, vol. LXI, Zurich, 1948, p. 280-302. En 1946 il donne à Bâle une des premières conférences sur les souffrances psychologiques consécutives aux persécutions nazies, et il intervient comme expert dans de nombreux procès intentés en réparation de ces crimes (Voir J. Pilliard, Eugène Minkowski, 1885-1972 et Françoise Minkowska, 1882-1950 : éclats de mémoire, Paris, L’Harmattan, 2009).

38. E. Minkowski, «Le contact humain », in Écrits cliniques, op. cit., p. 147, et, dans le même sens, 145.

39. J. Patočka, «L'homme spirituel et l'intellectuel » (1975), in Liberté et sacrifice, op. cit., p. 255 sq. 40. J. Patočka, « Séminaire sur l'ère technique » (1973), in Liberté et sacrifice, op. cit., p. 311 ; sur le sacrifice, voir encore L'Europe après l'Europe, Lagrasse, Verdier, 2007, p. 232. 